\title{
Quantitative EPMA of Nitrogen in Silicate Glasses
}

\author{
Anette von der Handt ${ }^{1}$, Celia Dalou ${ }^{1}$ \\ ${ }^{1}$ University of Minnesota, Dept. of Earth Sciences, Minneapolis (USA)
}

Nitrogen is the dominant gas in the Earth's atmosphere and the key to planetary habitability. However, exchange processes of nitrogen between deep Earth reservoirs (crust, mantle and core) and the surface (atmo-, hydro- and biosphere) are still not well understood. Accurate determination of nitrogen at low concentrations is important to place constraints on $\mathrm{N}$ partitioning during planetary differentiation and to address its role in the evolution of the early atmosphere.

The experimental determination of $\mathrm{N}$ partitioning and solubility at high pressure and temperature conditions requires the use of microbeam techniques due to the size of the experimental charges. In addition, silicate glasses at relatively high $\mathrm{fO}_{2}$ can have $\mathrm{N}$ concentration levels as low as a few tens of ppm.

Determination of ultra-light elements such as nitrogen by EPMA has consistently been fraught with problems due to its low fluorescence yield, resulting in low count rates and poor peak-to-background ratios [1, 2]. Accordingly, few studies on $\mathrm{N}$ in silicate glasses have been carried out by EPMA [3-7]. These studies unfortunately only report very limited information on analytical conditions, detection limits and uncertainties. One study [4] report a practical detection limit of $1500 \mathrm{ppm}$, limiting the compositional range that can be accurately constrained in samples, while another [7] achieves lower detection limits using a calibration curve approach limiting its application to very simple systems.

We developed an analytical protocol on our JEOL JXA-8900 at the University of Minnesota that resolves concentrations in silicate glasses down to a detection limit of $0.04 \mathrm{wt} \% \mathrm{~N}$ with a reasonable analysis time. Analytical errors were usually better than $3 \%$ for concentrations $>0.6 \mathrm{wt} \%$.

The strong curvature of the background for nitrogen on LDE monochromators demands application of advanced exponential background models, fit to wavelength scans. The presence of higher order metal lines close to the $\mathrm{N}$ peak has to be taken into account when choosing background positions. Conveniently, our electron microprobe has two spectrometers with LDE1 monochromators that are positioned opposite from each other, facilitating use of aggregate intensities to improve count rates while monitoring artifacts due to sample preparation that would result in inaccurate data.

Our analytical routine is split into multiple steps that take into account differences between unknowns and standard materials due to their different concentration levels. This helps to avoid dead time and pulse-height shift problems and to optimize individual background models. This approach was made possible by using the advanced options of the Probe for EPMA software.

In detail, each analytical session proceeded with the following steps:

1) Standards and unknowns were coated together in each analytical session.

2) Major elements were acquired at $15 \mathrm{kV}$ and $20 \mathrm{nA}$ on standards and unknowns.

3) Nitrogen was acquired on nitride standards at $10 \mathrm{kV}, 50 \mathrm{nA}$ with wide-set background positions. 
4) Nitrogen was acquired on unknowns at $10 \mathrm{kV}, 150 \mathrm{nA}$ with narrowly set background positions.

5) Exponential background models were applied, fitted to previously acquired wavelength scans.

6) Major element and nitrogen data were then combined in Probe for EPMA for each analysis point.

7) Nitrogen data were checked to make sure that spectrometers did not deviate from each other outside of their range of analytical precision.

8) Nitrogen intensities from both spectrometers were aggregated.

9) Final data processing used the PROZA matrix correction [8] and FFAST MACs [9].

References:

[1] M Raudsepp, Canadian Mineralogist 33 (1995), pp. 203-203.

[2] GF Bastin, HJM Heijligers, Scanning 13 (1991), pp. 325-342.

[3] M Roskosz et al., Geochimica et Cosmochimica Acta 70 (2006), pp. 2902-2918.

[4] M Roskosz et al. Geochimica et Cosmochimica Acta, 121 (2013), pp. 15-28.

[5] AA Kadik et al., Geochemistry International 49 (2011), pp. 429-438.

[6] AA Kadik et al., Geochemistry International 53 (2015), pp. 849-868.

[7] Y Li et al., Earth and Planetary Science Letters 41 (2015), pp. 218-228.

[8] GF Bastin et al., X囚Ray Spectrometry 27 (1998), pp. 3-10.

[9] CTJ Chantler, Nat. Inst. Stand. Techn., Gaithersburg, MD, (2005) http://physics.nist.gov/ffast
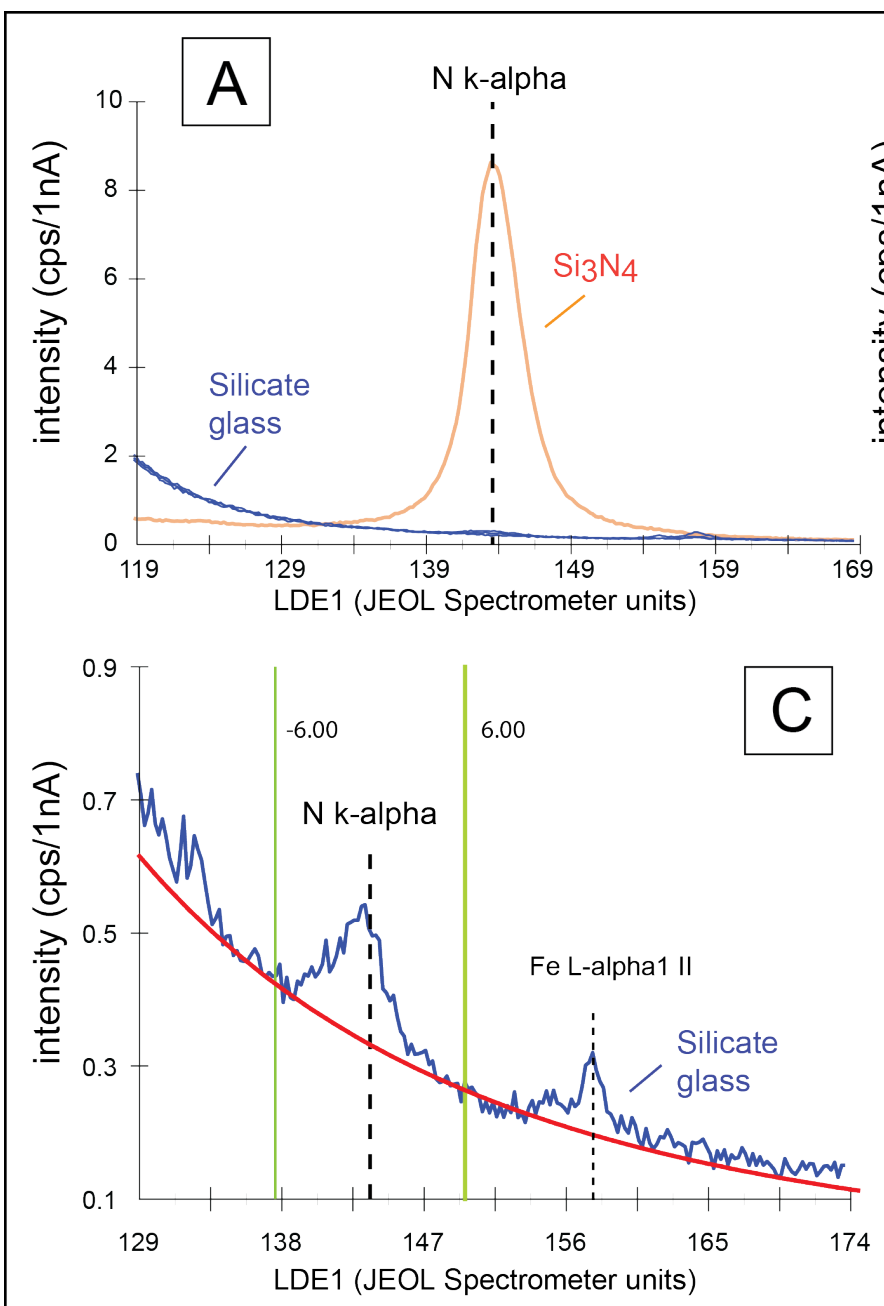

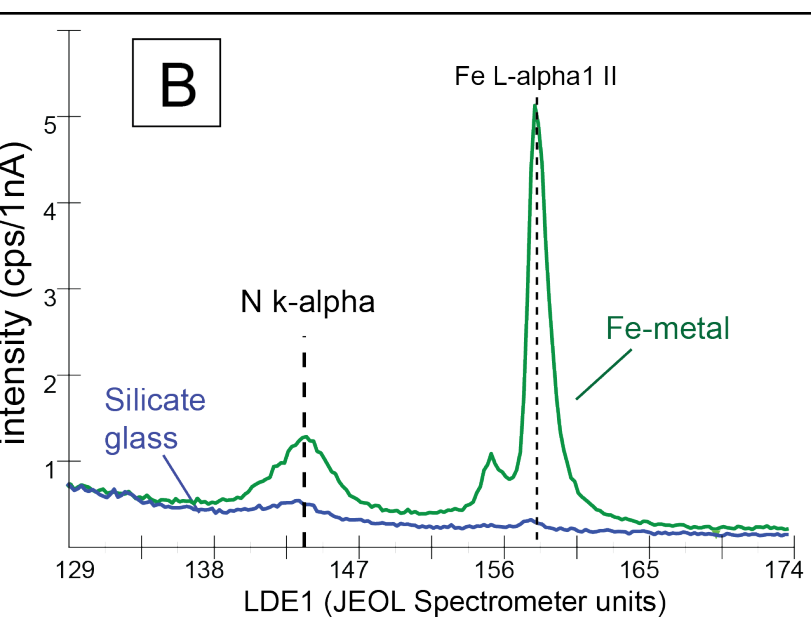

Figure 1. Nitrogen spectra recorded on LDE1 on various materials. A: Comparison of nitrogen spectra of Si3N4 and nitrogen-bearing silicate glasses. The background geometry differs between both materials. B: Position of higher order metal lines close to the $\mathrm{N}$ k-alpha peak, here iron that occurs in high abundance in our material, needs to be carefully recorded. C: Highly curved background geometry requires exponential background fits for accurate results. 